\title{
piRNA-14633 promotes cervical cancer cell malignancy in a METTL14-dependent m6A RNA methylation manner
}

Qi Xie ${ }^{1}$, Zhen $\mathrm{Li}^{1}$, Xiao Luo ${ }^{2}$, Dan Wang ${ }^{3}$, Yao Zhou ${ }^{3}$, Jingge Zhao ${ }^{4}$, Suhua Gao ${ }^{4}$, Yongguang Yang ${ }^{4}$, Wanying Fu ${ }^{4}$, Lingfei Kong ${ }^{1}$ and Tingyi Sun ${ }^{1 *}$ (D)

\begin{abstract}
Background: Cervical cancer (CC) is one of the most common gynecological tumors that threatens women's health and lives. Aberrant expression of PIWl-interacting RNA (piRNA) is closely related with a range of cancers and can serve as a tumor promoter or suppressor in proliferation, migration and invasion. In this study, the aim was not only to discover differential expression of piRNA in CC tissue (CC cells) and normal cervical tissue (normal cervical epithelium cells), but also to investigate the biological function and action mechanism of piRNA in CC.
\end{abstract}

Methods: The DESeq2 approach was used to estimate fold change in piRNA between CC tissue and normal cervical tissue. The relative expressions of piRNAs (piRNA-20657, piRNA-20497, piRNA-14633 and piRNA-13350) and RNA m6A methyltransferases/demethylases were detected using RT-qPCR. After intervention with piRNA-14633 and METTL14 expression, the viability of CaSki cells and SiHa cells was detected by CCK8. CC cell proliferation was detected by colony formation assay. Apoptosis rate and cell cycle were detected by flow cytometry. Transwell assay was performed to detect cell migration and invasion. EpiQuik m6A RNA Methylation Quantification Kit was used to evaluate m6A RNA methylation levels. Expression of methyltransferase-like protein 14 (METTL14), PIWIL-proteins and CYP1B1 were detected by RT-qPCR and western blot. The effect of piRNA-14633 on METTL14 was evaluated by a dual-luciferase reporter assay. The in vivo effects of piRNA-14633 on CC was assessed by nude mice experiments.

Results: piRNA-14633 showed high expression in CC tissues and cells, piRNA-14633 mimic (piRNA-14633 overexpression) promoted viability, proliferation, migration and invasion of CaSki cells and SiHa cells. Besides, piRNA-14633 mimic increased m6A RNA methylation levels and METTL14 mRNA stability. Results of dual luciferase reporter assays indicated that METTL14 was a directed target gene of piRNA-14633. Knockdown of METTL14 with siRNA attenuated proliferation, migration and invasion of CC cells. piRNA-14633 increased CYP1B1 expression, while silencing of METTL14 impaired its expression. The effect of piRNA overexpression on METTL14 expression has concentration-dependent characteristics. Results from in vivo experiment indicated that piRNA-14633 promoted cervical tumor growth.

Conclusion: piRNA-14633 promotes proliferation, migration and invasion of CC cells by METTL14/CYP1B1 signaling axis, highlighting the important role of piRNA-14633 in CC.

Keywords: Cervical cancer, piRNA-14633, Methyltransferase-like protein 14, m6A RNA methylation, Malignancy

\footnotetext{
*Correspondence: suntingyi99@163.com

1 Department of Pathology, Henan Provincial People's Hospital, People's

Hospital of Zhengzhou University; People's Hospital of Henan University, Zhengzhou, Henan 450003, People's Republic of China

Full list of author information is available at the end of the article
}

\section{Background}

Cervical cancer (CC) is the third common cancer in women worldwide, with 604,127 new cases and 341,831 deaths in 2020 [1]. High-risk human papillomavirus 
(HPV) infection is one of the main causes of CC. Currently, early-stage $\mathrm{CC}$ is treated with surgery, chemotherapy or radiotherapy; treatments for late-stage CC involves chemotherapy and radiotherapy, accompanied by poor prognosis $[2,3]$. Unfortunately, the higher recurrence rate is major obstacles to the long-term use of chemotherapy and radiotherapy for $\mathrm{CC}[4,5]$. It is therefore of great interest to search for novel markers of CC to provide new ideas and therapeutic targets for clinical examination, early diagnosis and prevention.

Piwi-interacting RNA (piRNA) was first identified in 2006 and is defined as a small non-coding RNAs that binds specifically to PIWI family proteins in testicular germ cells $[6,7]$. PiRNA is approximately 30 nucleotides in length and regulates transposon silencing and mRNA turnover, both of which are critical for gametogenesis and germ cell functions [8-11]. Recent studies have shown that piRNA is effectively expressed in both normal somatic cells and cancer cells [12]. PiRNA has also been shown to exhibit either pro-cancer or anticancer role by directly binding to PIWI proteins, highly conserved RNA-binding proteins [10]. In humans, so far four homologs have been identifed: PIWIL1, PIWIL2, PIWIL3, and PIWIL4. PiRNA can influence diverse processes in cancer cells, including apoptosis, proliferation, migration and invasion, by regulating gene expression at both transcriptional and post-transcriptional levels [1215]. Therefore, the aim of this study was to explore the effect and exact mechanism of piRNA in CC.

In recent years, emerging reports have indicated that epigenetic regulation plays a crucial role in the biological behaviors of cancer [16, 17]. N6-methyladenosine (m6A) of RNA, one of the most prevalent internal epigenetic regulations, has been involved in physiological and pathological processes of CC [18-20]. m6A RNA regulatory proteins can be divided into three classes: methyltransferases, demethylases and m6A-binding proteins. The methyltransferases, also known as writers and responsible for m6A formation, are composed mainly of methyltransferase-like 3 (METTL3), METTL14 and Wilms' tumor 1-associating protein (WTAP); demethylases, also known as erasers, catalyse the demethylation of m6A and are composed of fat mass and obesity-associated (FTO) and alkB homolog 5 (ALKBH5); m6A-binding proteins, also known as readers and determining the destiny of targeted RNAs, consists primarily of YT521-B homology (YTH) domain family proteins (YTHDF1/2/3) and YTH domain containing proteins (YTHDC1/2) [21-23]. The relationship between m6A RNA and piRNA have been proved in cardiac hypertrophy [24] and diffuse large B cell lymphoma [25].

However, it is unclear whether there is a relation exists between m6A RNA and piRNA in CC. Therefore, we aimed to investigate the expression level of piRNA14633 in CC and its correlation with prognosis to further explore its role in epitranscriptomic regulation in $\mathrm{CC}$ and its potential contribution to $\mathrm{CC}$ pathogenesis.

\section{Materials and methods \\ Patients and samples}

Formalin-fixed paraffin-embedded cervical cancer samples were obtained from the department of Pathology, Henan Province People's hospital. All patients did not receive radiotherapy, chemotherapy, targeted therapy and immunotherapy before surgery. All patients were diagnosed with colon cancer by HE (Hematoxylin-Eosin) staining. The research was approved by the Medical Ethics Committees of Henan Province People's hospital.

\section{RNA isolation, sequencing, and data analysis}

RNA from individual samples was isolated using the Universal RNA Kit (QIAGEN) and quantified using a Nanodrop spectrophotometer (Thermo Scientific, Waltham, MA, USA). All RNA samples were subjected to RNA integrity analysis using a Bio-Analyzer (Agilent Technologies, Santa Clara, CA, USA). Library preparation from polyA selected RNA and RNA-Seq was carried out by the DNA Sequencing. DESeq2 offers a comprehensive and general solution for gene-level analysis of RNA-seq data. Differentially expressed genes that were identified by both DESeq 2 .

\section{Cell culture}

Two cervical cancer cell lines Caski and Siha, as well as normal cervical endometrial cell lines End1/E6E7 and HUCEC, were purchased from Wuhan vector science Co.,Ltd (Dong $\mathrm{Hu}$ new technology development zone, Wuhan, Hubei, China). Dulbecco Modified Eagle's Medium (DMEM; Hyclone, South Logan, UT, USA) was employed to culture End1/E6E7 and HUCEC cells, while Caski and Siha cells were cultured in RPMI1640 medium containing at $37{ }^{\circ} \mathrm{C}$ in a humidified atmosphere of $5 \%$ $\mathrm{CO}_{2}$. All the mediums were contained with $10 \%$ fetal bovine serum (FBS; Hyclone, South Logan, UT, USA) and and $50 \mathrm{U} / \mathrm{ml}$ of penicillin/streptomycin.

\section{Vectors and transfection}

The piRNA-14633 mimic and the piRNA-14633 inhibitor oligonucleotides, as well as the corresponding negative control, were synthesized from Ribobio (Guangzhou, China). Caski and Siha cells were seeded in 6-well plate and cultured overnight for transfection. In accordance with the manufacturer's instructions, Lipofectamine 2000 (Invitrogen, Carlsbad, CA, USA) and equal amounts of oligo fragments were diluted by Opti-MEM/Reduced serum medium (Thermo Fisher Scientific, Waltham, MA, 
USA), respectively. Once the two solutions were mixed, the mixture was added into a 6-well plate. The medium was renewed $12 \mathrm{~h}$ after transfection. The cells were harvested $48 \mathrm{~h}$ after transfection.

\section{siRNA transfections}

Cells were transfected with pools of scrambled or target gene-specific siRNAs (100 nM) using Lipofectamine 2000 according to the manufacturer's instructions. The sequences of the siRNA against METTL14\#1 is as follow: GCAGCACCUCGA UCAUUUATT and the sequence of METTL14\#2 is as follow:GGAUGAAGGAGAGACAGA UTT.

The sequence of si-NC is as follow: UUCUCCGAACG UGUCACGUTT.

\section{RNA m6A quantification}

Total RNA was extracted via TRIzol (Invitrogen, CA, USA) as described below, and RNA quality was assessed by NanoDrop (Thermo Fisher Scientific, Waltham, MA, USA). The m6A modification level of total RNA was examined via EpiQuik m6A RNA Methylation Quantification Kit (P-9005; Epigentek Group Inc., Farmingdale, NY, USA) according to the instruction. Briefly, $200 \mathrm{ng}$ RNA accompanied with m6A standard were coated on assay wells, followed by capture antibody solution and detection antibody solution. The m6A levels were quantified colorimetrically by reading the absorbance of each well at a wavelength of $450 \mathrm{~nm}$ (OD450), and then calculations were performed based on the standard curve.

\section{RT-qPCR analysis and RNA stability assay}

Total RNA from cell lines and clinical tissues was extracted with Trizol reagent (Invitrogen), the reverse transcription reactions were conducted with oligo (dT) or specific miRNA/piRNA stem-loop RT primers using the Revert Aid First Strand cDNA Synthesis Kit (Thermo). Relative RNA levels determined by RT-qPCR were measured on the Light Cycler 480 II using the SYBR Green method. The primer sequences used are shown in Additional file 1: Table S1. For quantification of piRNAs, realtime qPCR were performed using the Bulge-LoopTM qPCR kit (RiboBio, Guangzhou, China) according to the manufacturer's protocol. U6 small nuclear RNA was employed as an internal control in both cell lines. All experiments were performed in three biological replicates. The relative expression of RNA was calculated as the power value $(2-\triangle \triangle \mathrm{Ct})$. For RNA stability assay: the cells were treated with a-Amanitin at $5 \mu \mathrm{g} / \mathrm{ml}$. After incubation for $0 \mathrm{~h}, 12 \mathrm{~h}, 4 \mathrm{~h}$ and $36 \mathrm{~h}$, the cells were collected and RNA was extracted for RT-qPCR described above.

\section{Cell viability and colony formation assays}

Cell viability was measured using the CCK- 8 kit. The cells were seeded in the 6-well culture dishes and incubated at $37{ }^{\circ} \mathrm{C}$ in humidified incubator for two weeks. Colonies were fixed and stained with crystal violet and the number of colonies was counted.

\section{Cell cycle and apoptosis assays}

The Cell Cycle Analysis Kit (Beijing 4A Biotech Co., FXP0311-100) and AnnexinV/PI apoptosis Kit (Beijing 4A Biotech Co., FXP018-100) were used to assess cell cycle distribution and apoptosis, respectively.

\section{Wound healing assay}

Caski and Siha cells were seeded in the 6-well plate $5 \times 10^{5} /$ well so as to form a cell monolayer overnight. The "wound line" was carefully created by a $200 \mu \mathrm{L}$ sterile plastic tip. Cells were cultured for $24 \mathrm{~h}$. Scratch-wound images at 0 and $48 \mathrm{~h}$ were captured using an Olympus IX71 microscope and wound-healing ability was calculated based on the relative cell-free area normalized to that in the $0 \mathrm{~h}$ image.

\section{The invasion and migration assays}

Invasion assay was done in a 24-well Millicell chamber. The $8-\mu \mathrm{m}$ pore inserts were coated with $30 \mu \mathrm{g}$ of Matrigel (BD Biosciences). Cells were added to coated filters in $200 \mu \mathrm{L}$ of serum-free medium in triplicate wells. $500 \mu \mathrm{L}$ medium containing $20 \%$ fetal bovine serum was added to the lower chamber as chemoattractant. After $48 \mathrm{~h}$ at $37{ }^{\circ} \mathrm{C}$ in a $5 \% \mathrm{CO}_{2}$ incubator, the Matrigel coating on the upper surface of the filter was removed. Cells that migrated through the filters were fixed with methanol, stained with $0.5 \%$ crystal violet, and photographed. Cell number on three random fields was counted. The migration assay was conducted in a similar fashion without coating with Matrigel.

\section{Western blot analysis}

Protein extracts from cells were prepared using detergent-containing lysis buffer. Total protein $(50 \mu \mathrm{g})$ was subjected to SDS-PAGE and transferred to PVDF membrane (Millipore). Antibodies against METTL14 (ab252562), PIWIL2 (ab151398) and PIWIL4 (ab111714) were from Abcam. Antibody against PIWIL1 (15,659-1-AP), CYP1B1 (18,505-1-AP) and $\beta$-actin $(60,008-1-$ Ig) were from Proteintech. Antibody against PIWIL3 (sc-398779) was from Santa. Then, the anti-rabbit or mouse horseradish peroxidase (HRP)-conjugated secondary antibody were conducted to incubate the membranes overnight at $4{ }^{\circ} \mathrm{C}$ and 
visualized with Immobilon Western Chemiluminescent HRP Substrate (Millipore).

\section{Luciferase reporter assay}

The METTL14 wild type (METTL14-WT) and METTL14 mutated type (METTL14-MUT) were constructed into luciferase pmirGLO reporter vector and followed by Dual-Glo Luciferase Assay system (NO. E608001, Sangon Biotech Co., Ltd. Shanghai, China). After $36 \mathrm{~h}$ transfection, the cells were lysed by passivelysis buffer. Firefly Luciferase (F-luc) and Renilla Luciferase(R-luc) of lysis were detected respectively.

\section{Animal experiments}

Animal experiments were carried out in compliance with approved protocols and guidelines from the Institutional Animal Care and Use Committee. To examine the effects of piRNA-14633 on subcutaneous xenograft growth, BALB/c nude mice (Beijing Vital River Laboratory Animal Technology) were subcutaneously injected with $0.1 \mathrm{~mL}$ of cell suspension containing $2 \times 10^{6}$ cells. Tumor volume $\left(\mathrm{mm}^{3}\right)$ was measured every 4 days using a Vernier caliper and calculated as $0.4 \times$ (short length) ${ }^{2} \times$ long length. Treatment was initiated when tumors reached a volume of $45-55 \mathrm{~mm}^{3}$ (day 16). The mice were randomly divided into 4 groups and received mi-NC, si-NC, mi-14633 and in-14633 by intratumor injection for 20 days. The mice were sacrificed, and the experiment was terminated at the end of 36 days. Tumors were isolated, weighed, and imaged.

\section{Statistical analysis}

All the experiments were performed 3 times at least. SPSS software (version 19.0, IBM Corp., Armonk, NY, USA) was used for statistical analysis of all the experimental data. GraphPad Prism (version 7, GraphPad Software, La Jolla, CA, USA) was used to determine the statistical results. All data are expressed as the mean \pm standard deviation (mean $\pm \mathrm{SD}$ ). The statistical analysis of the data from 2 groups was performed using a t-test. The comparisons of multiple groups were performed by one-way ANOVA and then an LSD- $t$ test. $\mathrm{P}<0.05$ was considered to be significant.

\section{Results}

\section{piRNA-14633 is overexpressed in cervical cancer}

In order to systematically study the role of piRNAs in cervical cancer, we first explored the expression profiles of five pairs of FFPE cervical cancer tissues and normal cervical tissues using the DESeq2 package and found some significant differentially expressed piRNAs with $\log 10($ FDR $)>0.2$ in cervical cancer tissues and normal cervical tissue (Fig. 1a). Figure 1b shows four significant differentially expressed piRNAs in cervical carcinoma tissue and normal cervical tissue. piRNA-14633/piRNA13350 were up-regulated and piRNA-20657/piRNA20497 were down-regulated in cervical carcinoma tissue compared with normal cervical tissue. Next, we validated the expression of these four piRNAs in normal cervical epithelial cells and cervical cancer cells by RT-PCR. We found that piRNA-14633 was significantly increased in cervical carcinoma cells compared to normal cervical epithelial cells (Fig. 1c). However, no significant difference was found in the expression of the other three piRNAs between cervical carcinoma cells and normal cervical epithelial cells. These results illustrate that piRNA-14633 may be a tumor promoter in cervical carcinoma.

The expression of piRNA-14633 was next explored to see if it was associated with the clinicopathological characteristics of the 94 cervical cancer patients. According to the results of RT-PCR, the 94 cervical carcinoma cases were divided into a piRNA-14633 low expression group $(\mathrm{n}=44)$ and a piRNA-14633 high expression group $(n=50)$. We found that increased levels of piRNA14633 were closely related to advanced TNM stage and increased tumor size (Table 1).

\section{piRNA-14633 promotes proliferation ability of cervical carcinoma cells}

Prior to studying possible roles of piR-14633, we investigated the expression of PIWIL1, 2, 3 and 4 in Caski and Siha cells, which are the prime proteins for biogenesis and functions of piRNAs. The results indicated that four homologs were expressed in CC cells (Fig. 2a),indicating their association with piRNA biogenesis and piRNA mediated regulatory functions. Next,we interfered with piRNA-14633 expression in Caski and Siha cells with mimic or inhibitor to to analyze its biological functions. We depleted piRNA-14633 expression using piRNA14633 inhibitor and increased piRNA-14633 expression using piRNA-14633 mimic in Caski and Siha cells. The efficiency of transfection was examined by RT-PCR. The results showed that the piRNA-14633 inhibitor reduced its expression and the piRNA-14633 mimic increased its expression in Caski and Siha cells (Fig. S1). As shown in CCK-8, inhibition of piRNA-14633 expression resulted in a dramatic decrease in the proliferation rate of Caski and $\mathrm{SiHa}$ cells, while increasing piRNA-14633 expression increased the proliferation rate of both cells (Fig. 2b). Similarly, the colony formation assay confirmed that piRNA-14633 increased the number of Caski and SiHa colonies (Fig. 2c and d).

Cycle and apoptosis of Caski and SiHa cells were examined by flow cytometry. As shown in Fig. 2e and f, inhibiting piRNA-14633 promoted cell cycle arrest in the G1 phase, and piRNA-14633 overexpression 

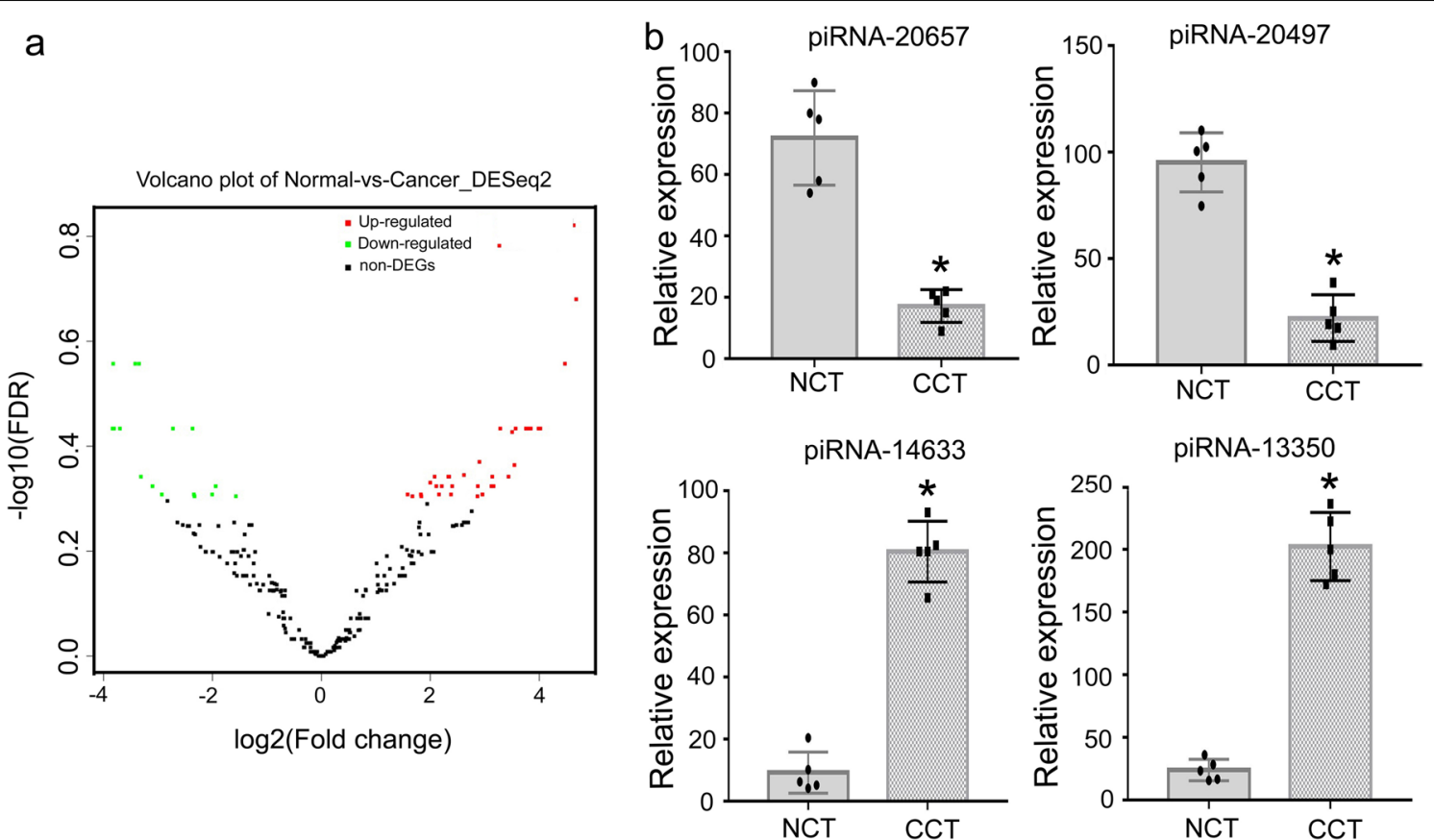

C
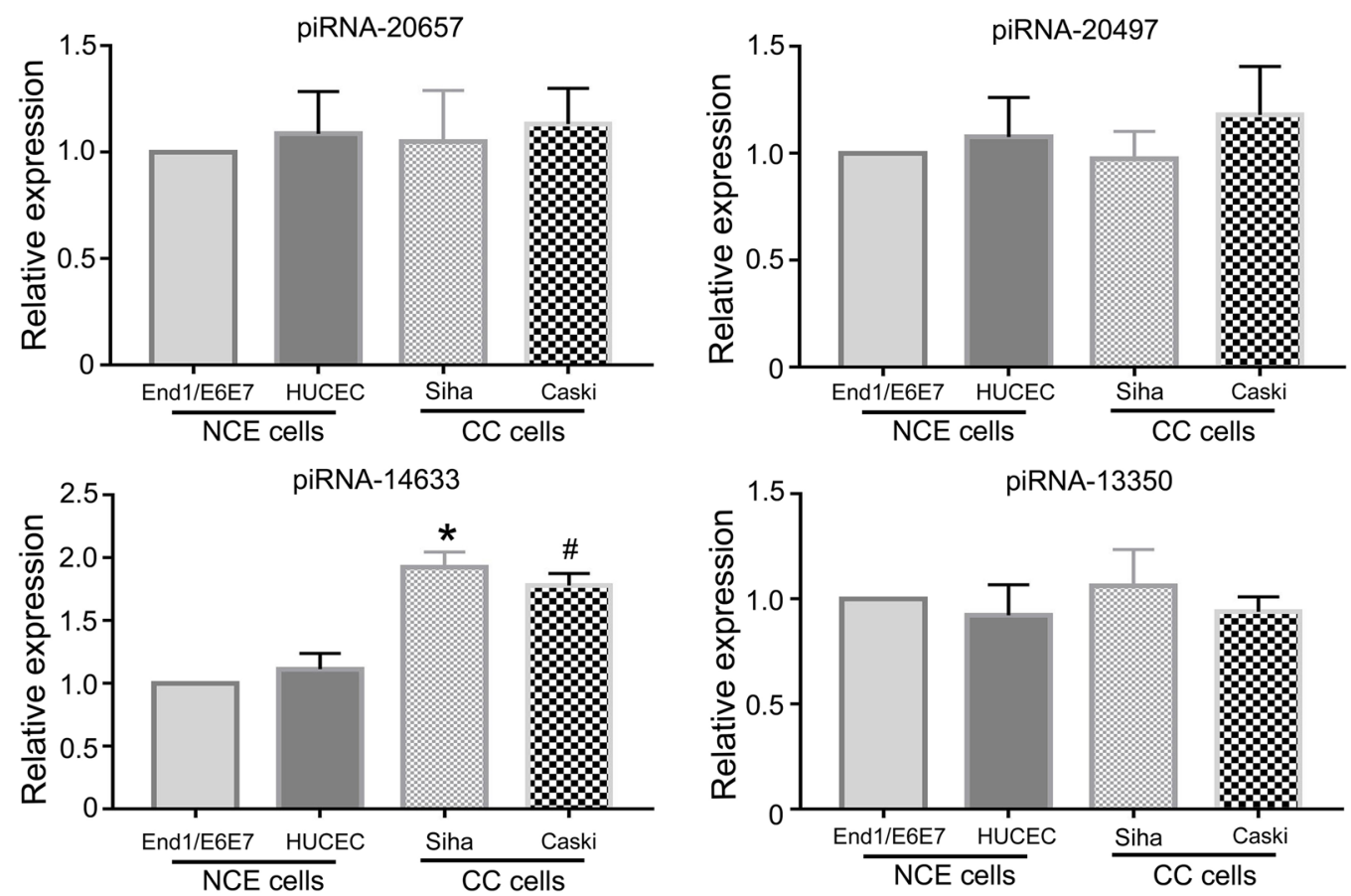

Fig. 1 piRNA-14633 is overexpressed in cervical cancer tissue and cell. a Folding changes and dispersion of piRNA in NCT and CCT were estimated using the DESeq method. $\mathbf{b}$ Four piRNAs expressions were compared in NCT and CCT. Data are presented as mean $\pm S D$. ${ }^{*} P<0.05$ vs. NCT. $\mathbf{c}$ Real-time PCR of four piRNAs expressions in CC cells (Siha and Caski) compared with NCE cells (End1/E6E7 and HUCEC). *P $<0.05$ vs. NCEC (End1/ E6E7 and HUCEC). ${ }^{"} P<0.05$ vs. NCEC (End1/E6E7 and HUCEC). All experiments are expressed mean \pm SD. NCT normal cervical tissue, CCT cervical cancer tissue, NCEC normal cervical endometrial cells, CCC cervical cancer cells

inhibited cell cycle arrest in the G1 phase. No significant apoptotic changes, however, were observed in Caski and SiHa cells for piRNA-14633 inhibitor or
piRNA-14633 mimic compared to negative control for inhibitor or negative control for mimic, respectively (Fig. $2 \mathrm{~g}$ and h). The above results indicated that 
Table 1 Correlation between the clinicopathological traits and piRNA-14633 expression in cervical carcinoma patients

\begin{tabular}{|c|c|c|c|c|}
\hline & \multirow[t]{2}{*}{ Total } & \multicolumn{2}{|l|}{$\mathrm{n} \%$} & \multirow[t]{2}{*}{$\mathbf{P}$} \\
\hline & & $\begin{array}{l}\text { Low expression } \\
\text { of piRNA-14633 }\end{array}$ & $\begin{array}{l}\text { High expression } \\
\text { of piRNA-14633 }\end{array}$ & \\
\hline \multicolumn{5}{|l|}{ Age } \\
\hline$\geq 55$ (years) & 55 & 25 (45.5\%) & $30(54.5 \%)$ & 0.755 \\
\hline$<55$ (years) & 39 & $19(48.7 \%)$ & $20(51.3 \%)$ & \\
\hline \multicolumn{5}{|l|}{ T stage } \\
\hline $\mathrm{T} 1-\mathrm{T} 2$ & 49 & $30(61.2 \%)$ & 19 (38.8\%) & $0.003^{*}$ \\
\hline T3-T4 & 45 & $14(31.1 \%)$ & 31 (68.9\%) & \\
\hline \multicolumn{5}{|l|}{ N stage } \\
\hline N1 & 42 & 27 (64.3\%) & 15 (35.7\%) & $0.002^{*}$ \\
\hline N2 & 52 & $17(32.7 \%)$ & 35 (67.3\%) & \\
\hline \multicolumn{5}{|l|}{ M stage } \\
\hline MO & 34 & $22(64.7 \%)$ & $12(35.3 \%)$ & $0.009^{*}$ \\
\hline M1 & 60 & $22(36.7 \%)$ & $38(63.3 \%)$ & \\
\hline \multicolumn{5}{|l|}{ Tumor size (cm) } \\
\hline$<4$ & 41 & 25 (61.0\%) & $16(39.0 \%)$ & $0.015^{*}$ \\
\hline$\geq 4$ & 53 & 19 (35.8\%) & $34(64.2 \%)$ & \\
\hline
\end{tabular}

T stage: Primary tumor stage; $\mathrm{N}$ stage: Regional lymph nodes stages; $\mathrm{M}$ stage: Distant metastasis stage

piRNA-14633 promoted proliferation ability and had no effects on apoptosis of cervical carcinoma cells.

\section{piRNA-14633 promotes migration/invasion abilities of cervical carcinoma cells}

Cell invasion and migration are known as the main indicators for assessing metastatic capacity. In contrast to the negative control inhibitor group, the trabecular surface of Caski and SiHa cells in the piRNA-14633 inhibitor group became significantly wider and migration weaker, as shown in Fig. 3a and b. While overexpression of piRNA-14633 induced narrower wound field of Caski and $\mathrm{SiHa}$ cells. Consistently, transwell results showed that piRNA-14633 overexpression increased the migration abilities of both cells and piRNA-14633 knockdown inhibited the migration abilities of both cells (Fig. 3c and d). We then tested the invasion abilities of cervical carcinoma cells by matrigel transwell assay. As shown in Fig. 3e and $f$, piRNA-14633 overexpression promoted invasion of Caski and SiHa cells compared to the mi-NC group, whilst cell invasion was attenuated in the piRNA14633 inhibitor group compared to the in-NC group. These results suggest that piRNA-14633 could promote the invasion and migration abilities of cervical carcinoma cells.
piRNA-14633 up-regulates m6A methylation and increases METTL14 expression in cervical carcinoma cells

Considering that piRNAs mainly act as important factors in m6A methylation, we first explored whether piRNA14633 affected the expression level of m6A methylation in cervical carcinoma cells. The results show that the total m6A abundance increased with the overexpression of piRNA-14633 and decreased with the down-regulation of piRNA-14633 (Fig. 4a and b).

RNA methyltransferases/demethylases play a crucial role in m6A methylation. We then examined the expression levels of METTL3, METTL14, WTAP, FTO and ALKBH 5 by RT-PCR in Caski and SiHa cells treated with piRNA-14633 mimic or inhibitor. The results showed that silencing piRNA-30473 could reduce the mRNA expression of METTL14, while overexpression of piRNA-30473 increased the mRNA expression of METTL14 (Fig. 4cf). But the mRNA expressions of other four genes were not significantly different in cells when in response to piRNA-14633 inhibitor or piRNA-14633 mimic, when compared to negtive control inhibitor or negtive control mimic, respectively. These results indicated that piRNA14633-mediated upregulation of m6A methylation levels might be relevant to to increased METTL14 expression in cervical carcinoma cells.

\section{METTL14 is a target of piRNA-14633 in cervical carcinoma cells}

To verify whether piRNA-14633 targets METTL14 in cervical carcinoma cells, we predicted the binding site between piRNA-14633 and 3'UTR of METTL14 (mRNA 5'-3', 4364-4392) by bioinformatics software (Fig. 5a). We then performed a dual-luciferase reporter assays by constructing wildtype (WT) and mutant (MUT) dualluciferase reporter vectors of METTL14 according to the potential binding sites of piRNA-14633 and METTL14. Figure $5 \mathrm{~b}$ exhibites the wild/mutant-type 3'UTR of METTL14 luciferase pmirGLO reporter vector. Translation of the luciferase reporter gene showed that silencing of piRNA-14633 resulted in a decrease in luciferase activity of the reporter construct carrying WT METTL14 relative to the inhibitor of the negative control, while there was no significant change in luciferase activity of the MUT reporter (Fig. 5c and d). Similarly, piRNA-14633 overexpression caused a increase in the luciferase activity of the reporter construct carrying the WT METTL14 relative to the negtive control minic, whereas the luciferase activity of MUT reporters was not significantly changed (Fig. $5 \mathrm{c}$ and d). We also confirmed that the effect of piRNA-14633 on METTL14 mRNA expression has concentration-dependent characteristics (Fig. 5e). In clinical specimens, we found that the expression of 


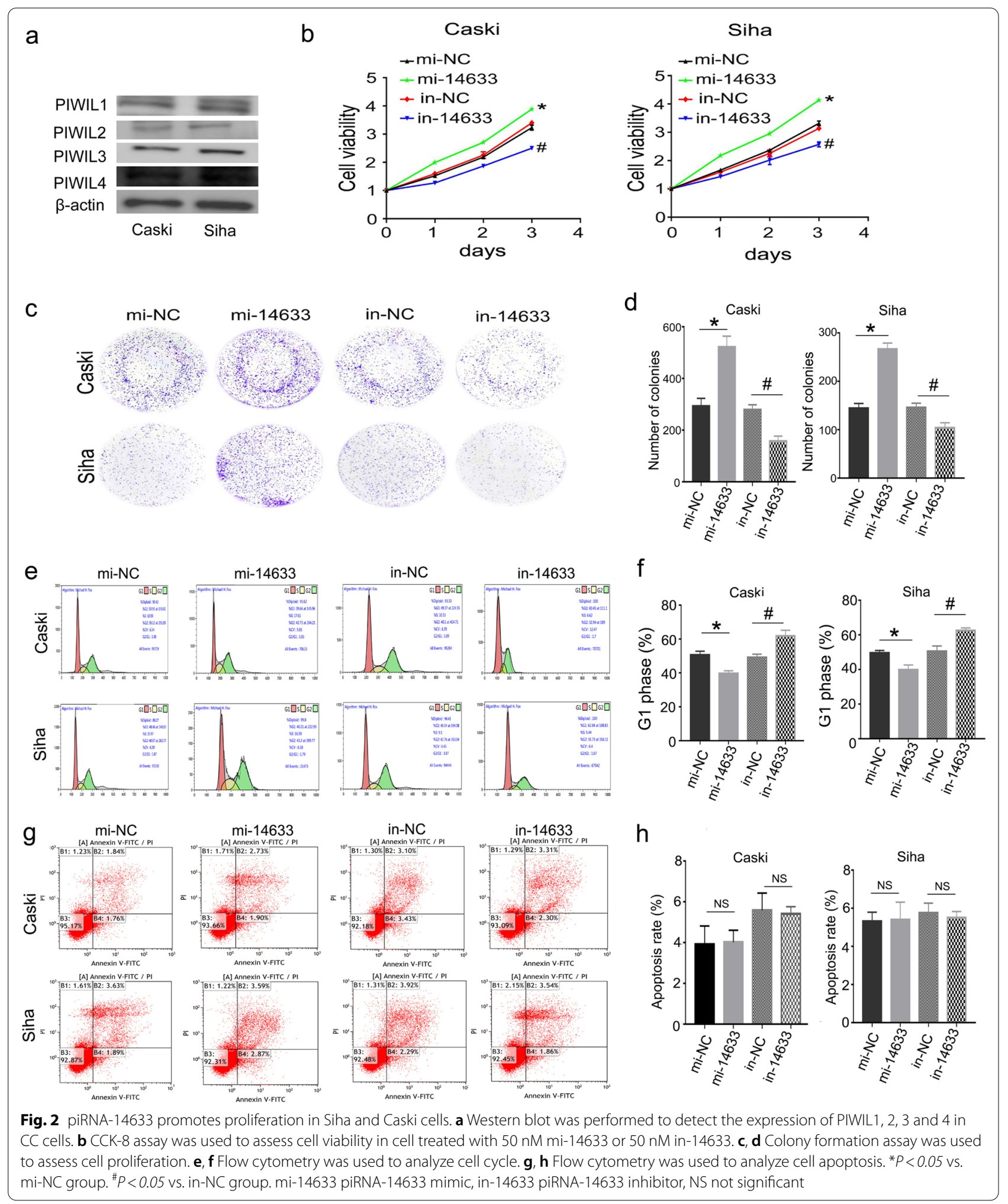



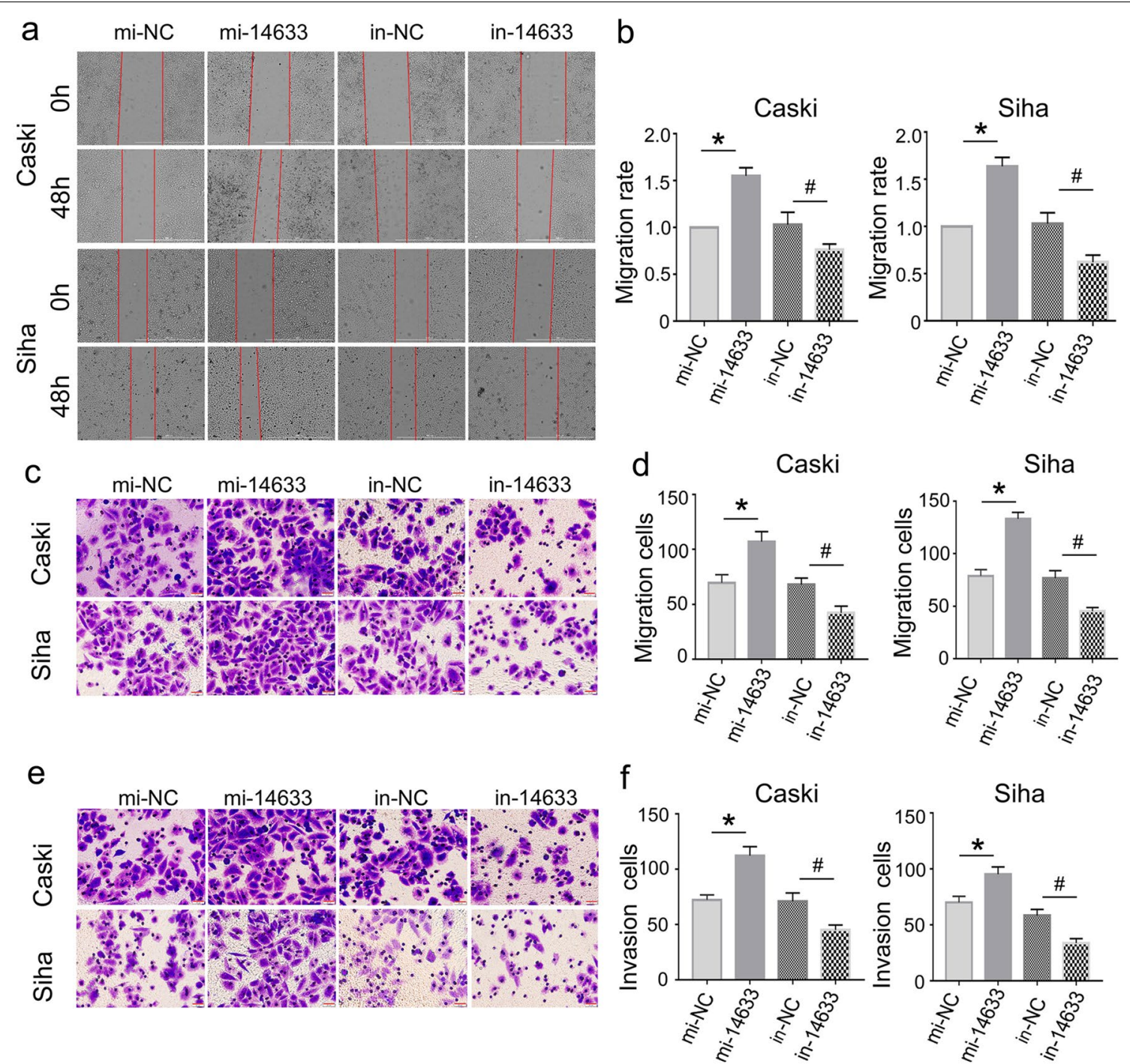

Fig. 3 piRNA-14633 accelerates migration/invasion abilities of Siha and Caski cells treated with $50 \mathrm{nM}$ mi-14633 or 50 nM in-14633. a, b Wound healing assay was used to analyze the migration capabilities of two cervical cancer cells. Scale bars, $1000 \mu \mathrm{m}$. $\mathbf{c}, \mathbf{d}$ Transwell assay was applied to measure migration abilities of Siha and Caski cells. Scale bars, $20 \mu \mathrm{m}$. e, f Transwell assay was applied to measure invasion abilities of Siha and Caski cells. Scale bars, $20 \mu$ m. ${ }^{*} P<0.05$ vs. mi-NC group. ${ }^{\#} P<0.05$ vs. in-NC group. mi-14633 piRNA-14633 mimic, in-14633 piRNA-14633 inhibitor

piRNA-14633 showed a strong positive correlation with METTL14 mRNA levels (Fig. 5f). The above results indicate that piRNA-14633 binds specifically to METTL14 in cervical carcinoma cells.

\section{piRNA-14633 elevates METTL14 protein expression by increasing stability of its mRNA in cervical carcinoma cells}

To further study the relationship between piRNA14633 and METTL14 in cervical carcinoma cells, we inhibited new mRNA synthesis of cervical carcinoma cells by a-Amanitin. By RT-PCR, we found that piRNA14633 overexpression increases half-life of METTL14 and piRNA-14633 knockdown decreases half-life of METTL14 in cervical carcinoma cells (Fig. 6a-d). In the next step, we examined protein expression of METTL14 in Caski and SiHa cells treated with piRNA14633 overexpression or knockdown. Western blot assays showe that piRNA-14633 overexpression could upregulate the expression of METTL14 (Fig. 6e and f). We also found that piRNA-14633 correlated positively with METTL14 mRNA expression in clinical FFPE specimens (Fig. 6g). These results suggest that piRNA14633 increases stability of METTL14 mRNA, which then elevated METTL14 protein expression in cervical carcinoma cells. 


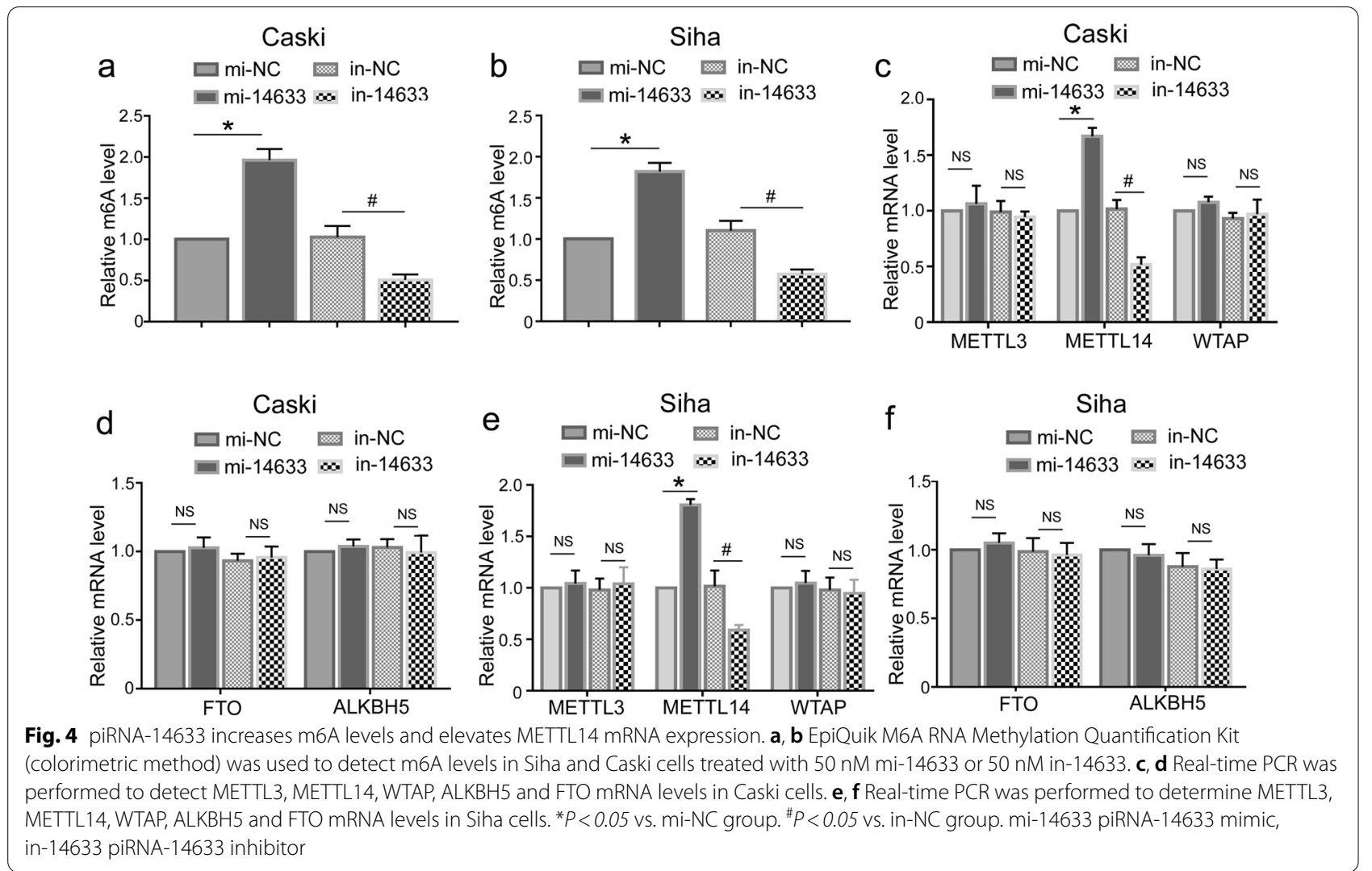

a

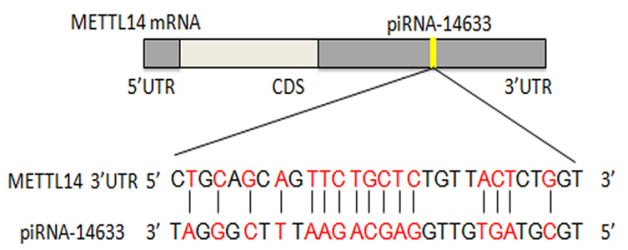

Siha

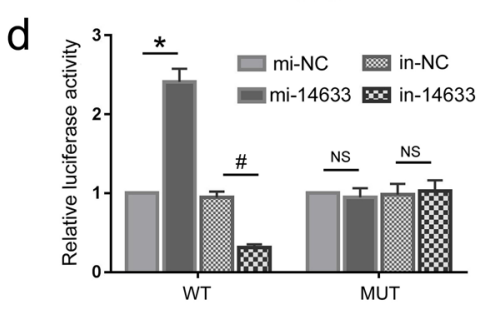

b
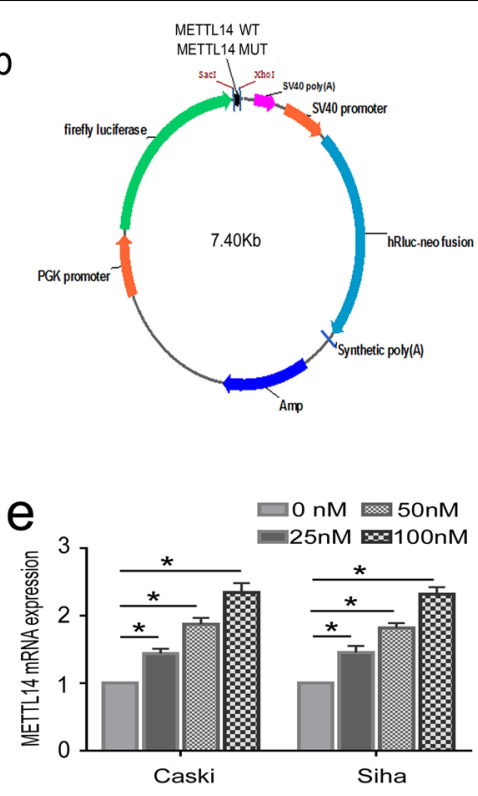

C
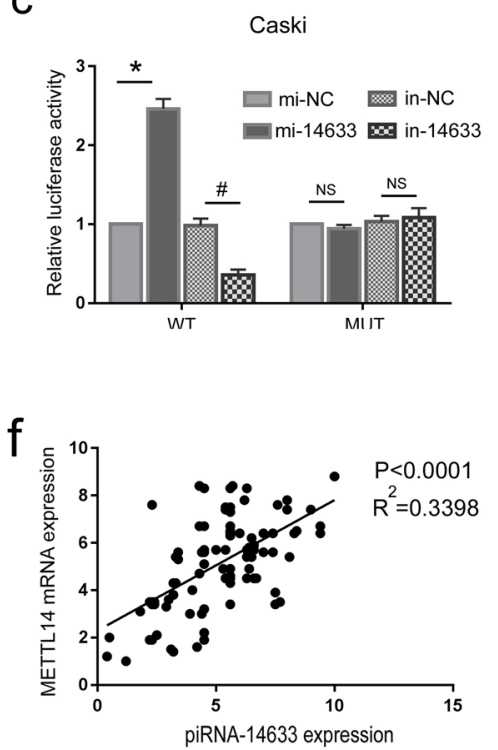

Fig. 5 METTL14 is a target of piRNA-14633 in Siha and Caski cells. a Diagram of the interaction between piRNA-14633 and the 3'UTR of METTL14. b Wild/mutant-type 3'UTR of METTL14 luciferase pmirGLO reporter vector. c, d Measurement of relative luciferase activity in Siha and Caski cells transfected with METTL14 -wild type or mutant-type, with $50 \mathrm{nM}$ mi-14633 or $50 \mathrm{nM}$ in-14633. e Real-time PCR was performed to detect the effect of piRNA-14633 ( $0 \mathrm{nM}, 25 \mathrm{nM}, 50 \mathrm{nM}$ and $100 \mathrm{nM})$ on METTL14 mRNA expression $\mathbf{f}$ The correlation of piRNA-14633 with METTL14 expression in clinical FFPE specimens. ${ }^{*} P<0.05$ vs. mi-NC group. ${ }^{\#} P<0.05$ vs. in-NC group. mi-14633 piRNA-14633 mimic, in-14633 piRNA-14633 inhibitor, NS not significant 


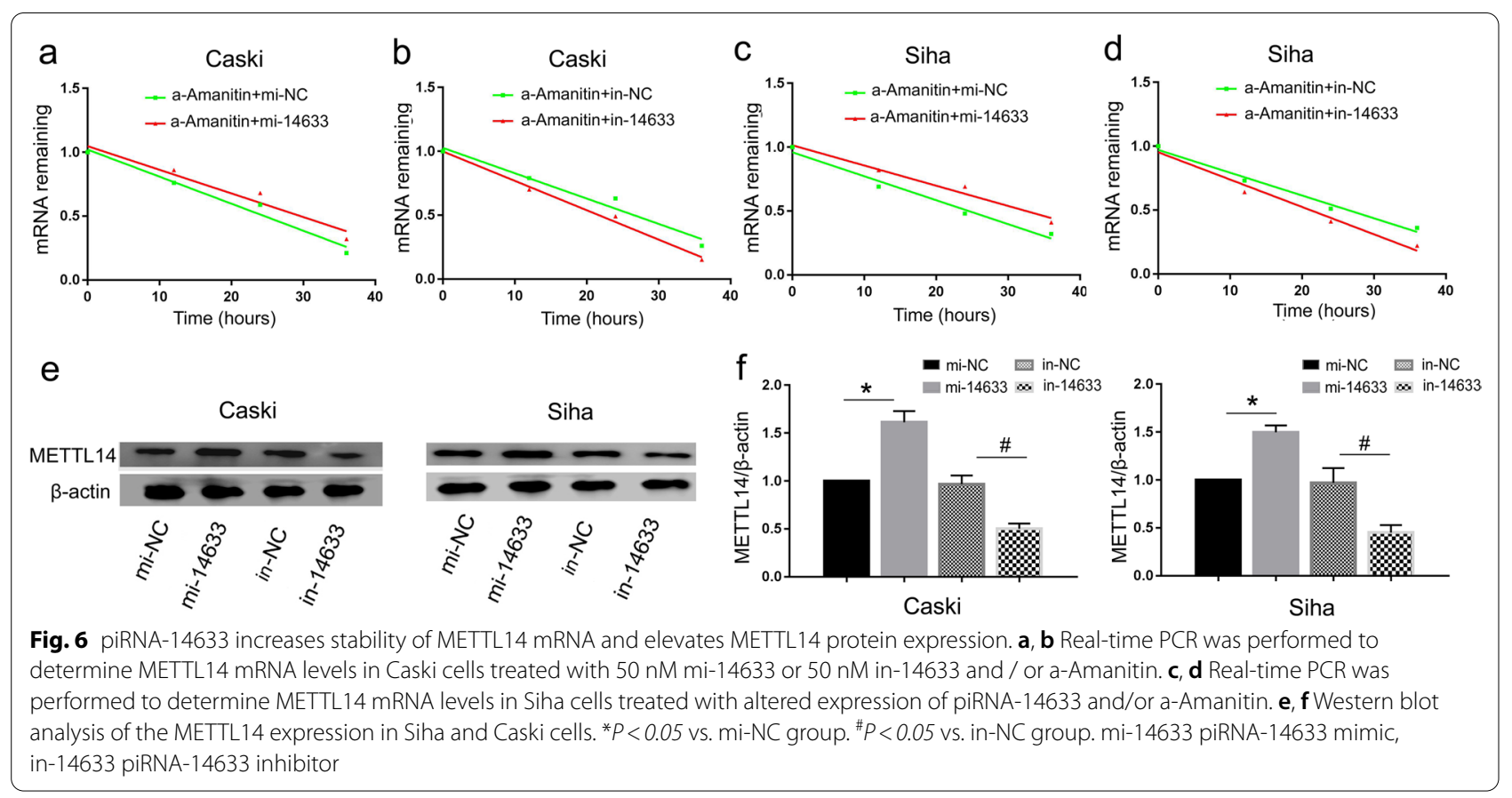

\section{a}

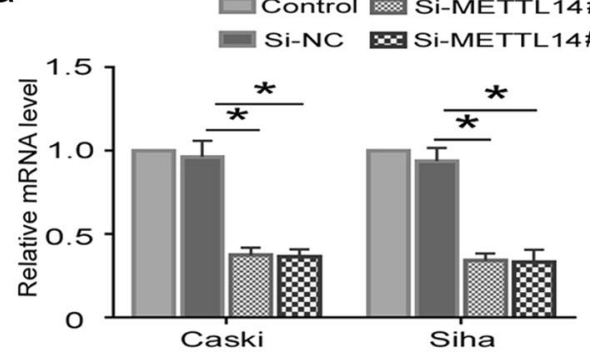

C

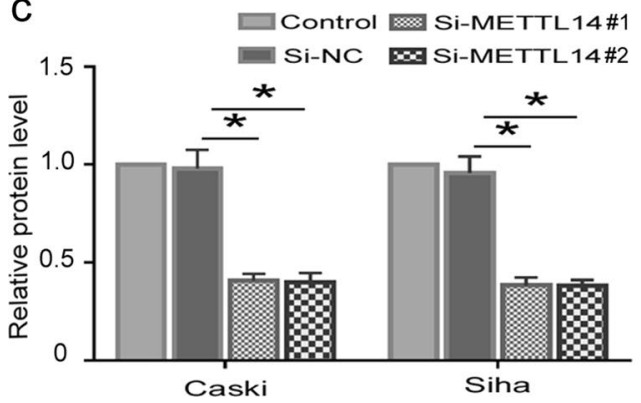

b
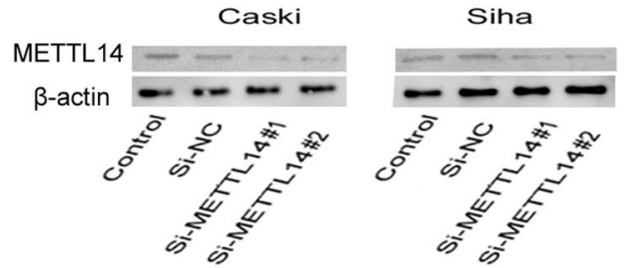

d

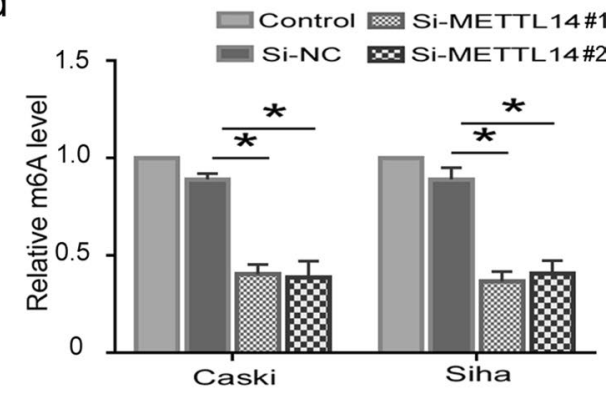

Fig. 7 METTL14 knockdown effectively attenuates m6A levels in Siha and Caski cells. a Real-time PCR detection of METTL14 expression in Siha and Caski cells after transfection with $50 \mathrm{nM}$ si-NC or $50 \mathrm{nM}$ si-METTL14\#1/2. b, c Western blot was performed to detect the expression of METTL14 in Siha and Caski cells after transfection with si-NC or si-METTL14\#1/2. d EpiQuik M6A RNA Methylation Quantification Kit (Colorimetric assay) was used to detect m6A levels in Siha and Caski cells. ${ }^{*} P<0.05$ vs. si-NC group. Si-NC siRNA-negtive control, si-METTL14\#1/2 siRNA-METTL14\#1/2

piRNA-14633 promotes proliferation ability of cervical carcinoma cells by increasing m6A modification

To further study the role of METTL14 in piRNA14633-mediated cervical cancer cells, we knocked down
METTL14 expression using small interference RNA (siMETTL14). RT-PCR and Western blot analysis showed that both si-METTL14 \#1 and si-METTL14 \#2 reduced its mRNA and protein levels in Caski and Siha cells 
(Fig. 7a-c). By Epiquik m6A RNA measurement quantifcation kit, we found that si-METTL14 decreased m6A methylation activity in cervical carcinoma cells (Fig. 7d). CCK-8 and colony formation assays showed that piRNA14633 promoted the proliferation of cervical carcinoma cells and that si-METTL14 could block the promotion effect (Fig. 8a-c). Furthermore, overexpression of piRNA-14633 inhibited cell cycle arrest in the G1 phase, which could be partly reverted by METTL14 silencing (Fig. 8d and e). These results demonstrated that piRNA14633 could enhance the proliferation ability of cervical carcinoma cells by increasing m6A methylation activity.

\section{piRNA-14633 promotes migration/invasion abilities of cervical carcinoma cells by METTL14-regulated m6A modification}

Wound healing assays then showed that piRNA-14633 tended to increase migration of cervical carcinoma cells, which were reversed by si-METTL14\#1 and siMETTL14\#2 (Fig. 9a and b). We also performed transwell assays and noticed that piRNA-14633 promoted the migration and invasion abilities of cervical carcinoma cells, while silencing of METTL14 impaired these phenotypes (Fig. 9c-f). This results provides some evidence for the existence of piRNA-14633/ METTL14/ m6A modification axis in cervical carcinoma cells.

\section{CYP1B1 may be target of METTL14-mediated m6A methylation regulated by piRNA-14633}

Previous studies have elucidated that CYP1B1 is critical downstream target of METTL14 in caner cells [26]. Of note, the secondary structures of CYP1B1 show a higher confidence m6A methylation sites [26]. We therefore hypothesized that METTL14/ CYP1B1 pathway might be involved in piRNA-14633-induced CC cell metastasis. By RT-PCR and Western blot, we reach the conclusion that piRNA-14633 promotes CYP1B1 expression by METTL14-mediated m6A methylation (Fig. 9g and h), suggesting that CYP1B1 may be a novel candidate direct
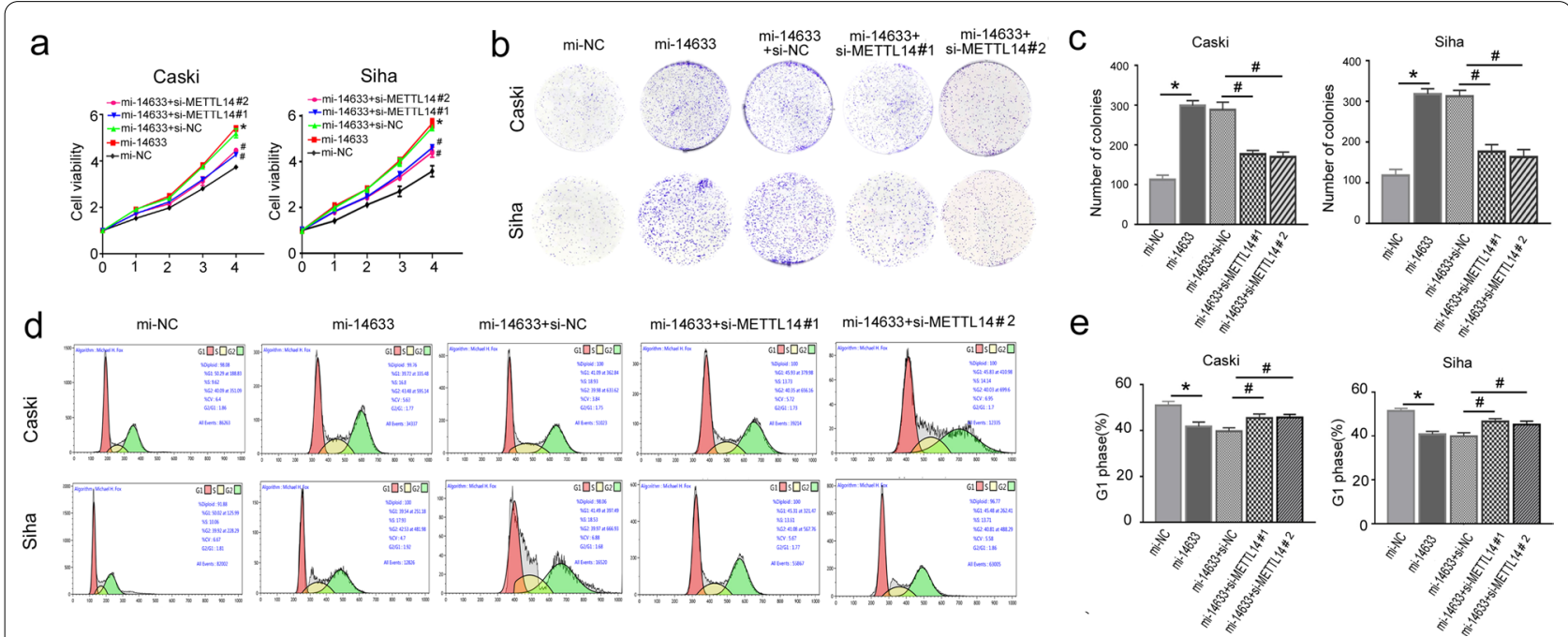

Fig. 8 piRNA-14633 promotes proliferation of cervical cancer cells via METTL14 binding. a CCK-8 assay was used to assess viability in cells transfected with $50 \mathrm{nM}$ mi-14633 or co-transfected with $50 \mathrm{nM}$ si-METTL14\#1/2. b, c Colony formation assay was used to assess cell proliferation. d, e Flow cytometry was used to analyze cell cycle. ${ }^{*} P<0.05$ vs. mi-NC group. ${ }^{\#} P<0.05$ vs. mi-14633 + si-NC group. mi-14633 piRNA-14633 mimic, in-14633 piRNA-14633 inhibitor, si-NC siRNA-negtive control, si-METTL14\#1/2 siRNA-METTL14\#1/2

(See figure on next page.)

Fig. 9 piRNA-14633 contributed to tumorigenesis of Siha and Caski cells via METTL14/CYP1B1 axis. a and b Wound healing assay was used to analyze the migration capabilities of two cervical cancer cells transfected with $50 \mathrm{nM}$ mi-14633 or co-transfected with $50 \mathrm{nM} \mathrm{si-METTL14 \# 1/2.}$ Scale bars, $1000 \mu \mathrm{m}$. c and d Transwell assay was applied to measure migration abilities of Siha and Caski cells. Scale bars, $20 \mu \mathrm{m}$. e and f Transwell assay was applied to measure invasion abilities of Siha and Caski cells. $g$ and $\mathrm{h}$ RT-PCR and Western blot waere used to analyze the expressions of CYP1B1 in two cervical cancer cells transfected with $50 \mathrm{nM}$ mi-14633 or co-transfected with $50 \mathrm{nM}$ si-METTL14\#1/2. Scale bars, $20 \mu \mathrm{m} .{ }^{*} P<0.05$ vs. mi-NC group. ${ }^{~} P<0.05$ vs. mi-14633 + si-NC group. mi-14633 piRNA-14633 mimic, in-14633 piRNA-14633 inhibitor, si-NC siRNA-negtive control, si-METTL14\#1/2 siRNA-METTL14\#1/2 

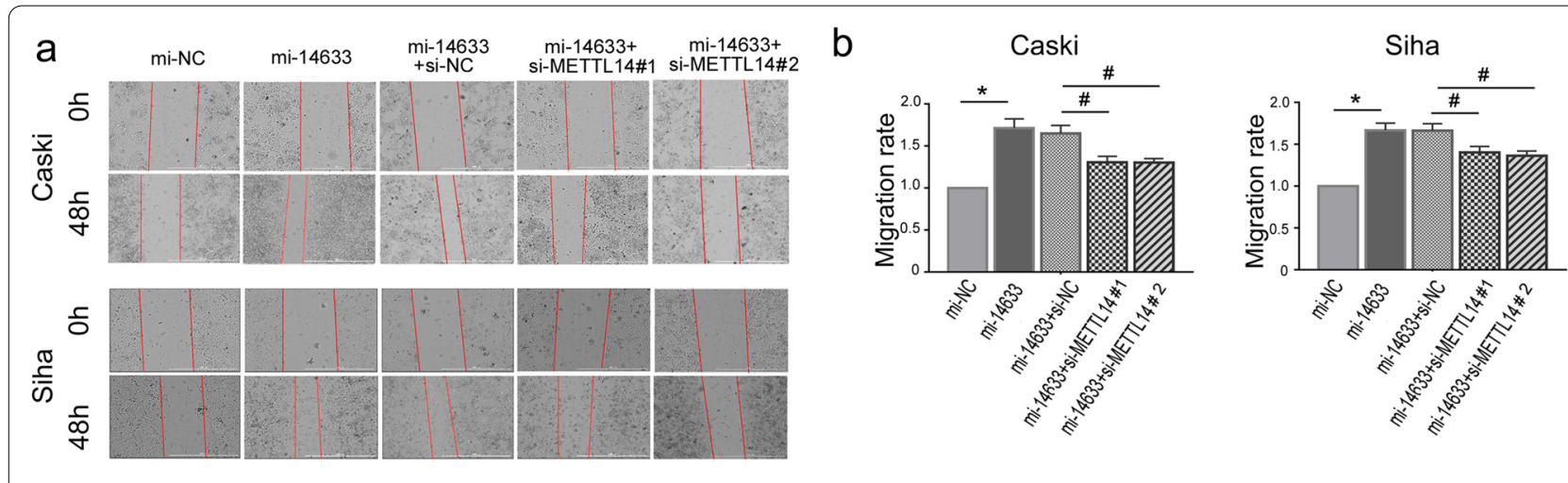

C

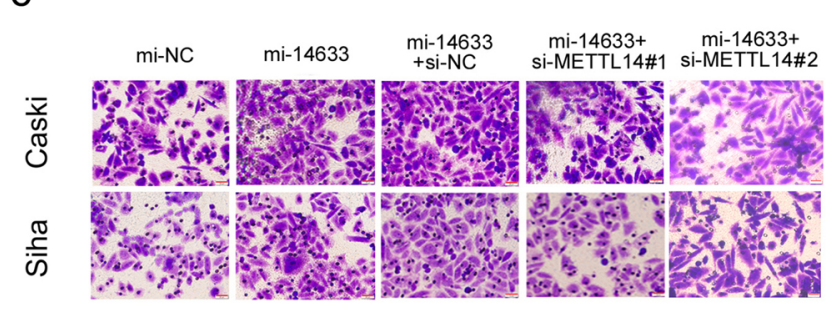

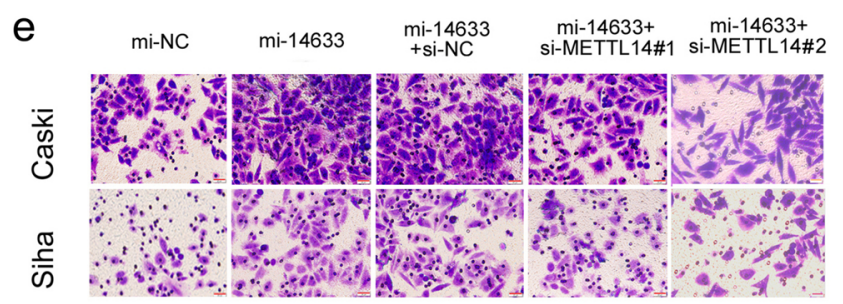

g

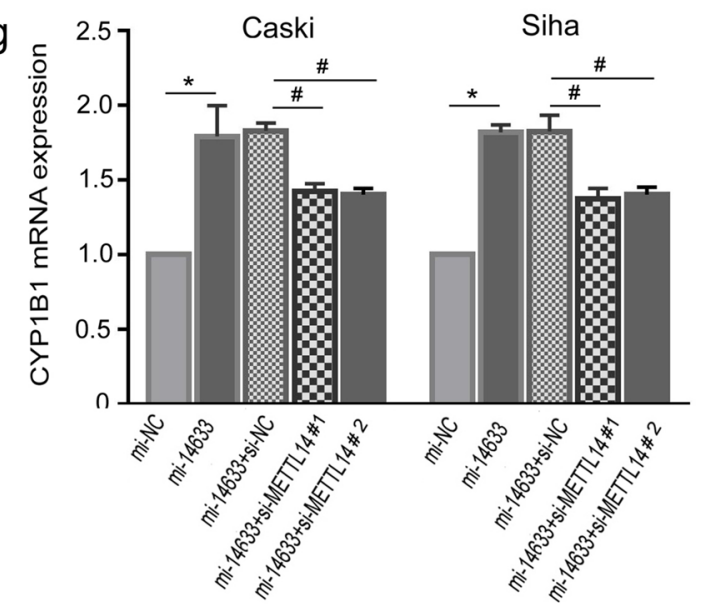

d

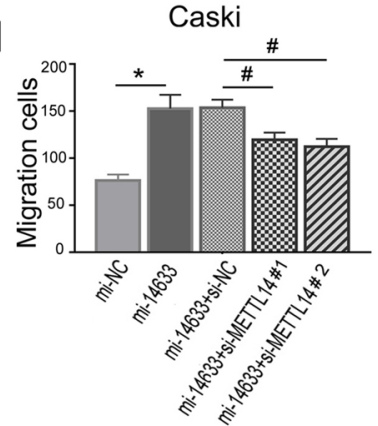

f
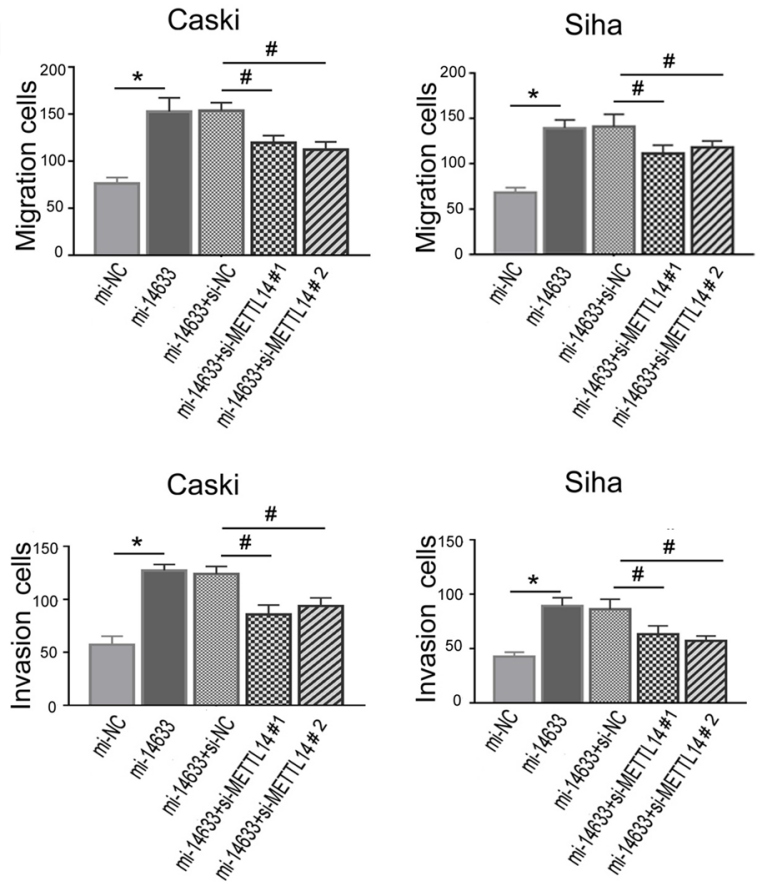

Sina

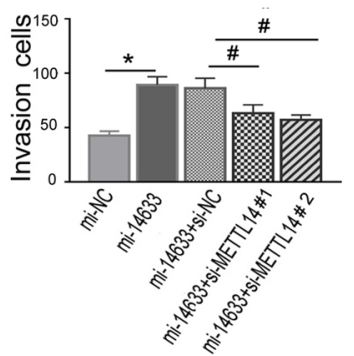

Fig. 9 (See legend on previous page.) 
target of METTL14-mediated m6A methylation regulated by piRNA-14633 in CC cells.

\section{piRNA-14633 promotes the growth of cervical carcinoma cells in vivo}

To further explore the effect of piRNA-14633 on cervical carcinoma in vivo, we a established xenograft mouse model by injecting equal amounts of Caski cells into the nude mice (3 mice in each group). Caski-derived tumors were treated with mi-14633 or in-14633 for 20 days. We then sacrificed the mice and found that piRNA14633 promoted tumor growths (Fig. 10a). piRNA14633expression had no impact on body weights of mice (Fig. 10b). The weight of tumors in the mi-NC group were lighter than that in the piRNA-14633 overexpression group (Fig. 10c). Similarly, the tumour weight of the piRNA-14633 knockdown group were lighter than that in the in-NC group (Fig. 10c). The aforementioned findings demonstrate that piRNA-14633 promoted tumor growth in cervical carcinoma.

\section{Discussion}

To date, over 20,000 piRNAs have been identified in eukaryotic genome. Aberrant expression of piRNAs has been demonstrated in a variety of cancers, including diffuse large B-cell lymphoma, colorectal cancer, lung cancer and other cancers[27-30]. Besides, piRNA has been reported to play a protumorigenic or antitumorigenic role by regulating some signaling molecules and signaling pathways[28]. Recent studies have already proved that piRNA-651 is highly expressed in lung cancer and promotes proliferation, invasion and metastasis by regulating expression of caspase-3, Bax and cyclinD 1[31]. piRNA-823 was found by Ding et al. to stimulate the growth of luminal breast cancer cells by regulating cancer stem cells[32]. In this study, we have confirmed the high expression rate of piRNA-14633 in CC cells and tissues.
We also found that overexpression of piRNA-14633 promotes proliferation, migration and invasion of Caski and Siha cells. piRNA-14633 overexpression induced G2/M arrest, regardless of apoptosis. Contrastingly, down-regulation of piRNA-14633 inhibits cells proliferation, migration and invasion, as well as induces G1 arrest. Using a subcutaneous tumour model, we demonstrated that piRNA-14633 contributes to the growth of the CC in vivo. The results illustrate that piRNA14633 may be a new regulator and a novel target for CC intervention and treatment.

The piRNA was indicated to be significantly associated with DNA methylation in the mammalian cells [33-35]. Specifically, through a proteomic approach, Zoch et al. reported that piRNA is critically involved in DNA methylation of the transposable elements by regulating SPOCD1 expression [34]. piRNA was also shown by $\mathrm{Fu}$ et al. to have the ability to promote DNA methylation of genes encoding non-translocatable related proteins [33]. In addition to their involvement in DNA methylation, piRNA has also been reported to be closely related to RNA methylation [36]. Cardiac hypertrophy-associated piRNAs have been shown to induce cardiomyocyte hypertrophy and poor cardiac remodeling by blocking m6A RNA methylation of PARP10 [24]. Han et al. proved that piRNA-30473 contributes to the progression of diffuse large B-cell lymphomaby increasing WTAP mRNA stability and enhancing HK2 m6A level [25]. In recent years, there have been many reports about the role of m6A RNA methylation in CC development and progression [20, 37, 38]. Ma et al. systematically analyzed the expression and clinical prognostic role of $13 \mathrm{~m} 6 \mathrm{~A}$ RNA methylation regulators in CC by cancer genome mapping [39]. Zou et al. showed that FTO contributes to proliferation and migration of $\mathrm{CC}$ and the cancer-promoting function of FTO in CC is dependent on m6A RNA demethylase [40]. In addition, increased METTL3 levels has been found in CC
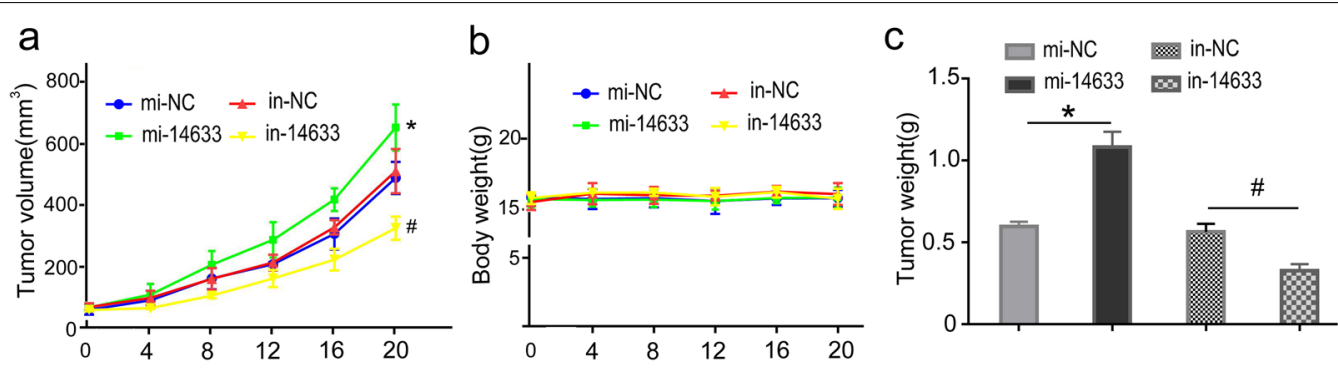

Fig. 10 piRNA-14633 promotes the growth of Caski-derived tumor xenografts in Balb/c nude mice. a Caski-derived tumors were treated with $50 \mathrm{nM}$ mi-14633 or $50 \mathrm{nM}$ in-14633 for 20 days. The volume of subcutaneous xenografts in nude mice was recorded every four days. $\mathbf{b}$ Tumor volumes in four different groups. $\mathbf{c}$ Body weights of mice from four different groups. ${ }^{*} P<0.05$ vs. mi-NC group. ${ }^{\#} P<0.05$ vs. mi- $14633+$ si-NC group. mi-14633 piRNA-14633 mimic, in-14633 piRNA-14633 inhibitor 
tissue and accelerated the CC carcinogenesis and aerobic glycolysis via up-regulating m6A RNA content [18]. However, there are no reports on relationship of piRNA expression and m6A RNA in CC.

Here, we identified that piRNA-14633 increases m6A RNA methylation in Caski and Siha cells. By bioinformation analysis and dual-luciferase reporter assays, we confirmed that piRNA-14633 specifically targets the 3'-UTR of METTL14. Besides, it was found that piRNA-14633 increased the expression of METTL14 by enhancing its mRNA stability. In clinical specimens, the expression of piRNA-14633 showed a strong positive correlation with METTL14 mRNA levels. To further demonstrate the role of METTL14 in m6A RNA methylation and tumorigenicity of $\mathrm{CC}$, we decreased METTL14 expression by siRNA technology. Downregulation of METTL14 weakened m6A RNA methylation levels induced by piRNA-14633. Si-METTL14 could counteract the promoting effect of piRNA-14633 on the proliferation, migration and invasion of cervical cancer cells, suggesting that piRNA-14633 accelerates cervical cancer progression by inducing METTL14mediated m6A methylation. Of course, more work needs to be done to identify $\mathrm{m} 6 \mathrm{~A}$ reader protein and METTL14 mediated m6A methylated target gene transcripts in piRNA-14633-treated CC cells. Studies have reported that CYP1B1 was expressed in the majority of the cervical cancer samples $(91 / 100,91.0 \%)$ but not in normal healthy cervical samples [41]. Our work speculates a unrecognized piRNA-14633-METTL14CYP1B1 signaling axis in CC cells. By directly binding to METTL14, piRNA-14633 targets METTL14 through the specific binding domain and subsequently stabilizing the expression and its downstream target such as CYP1B1. Hence, we therefore hypothesized that the METTL14/CYP1B1 pathway might be involved in piR14633-induced CC cell metastasis.

In conclusion, recent studies not only reported higher levels of piRNA-14633 in CC than in normal tissues, but also revealed that piRNA-14633 increased m6A RNA methylation and thus contributed to tumorigenicity of CC by specifically targetting the 3'-UTR of METTL14 and thereby triggering corresponding signaling cascades (Fig. 11). These findings indicate that the piRNA-14633/ METTL14/CYP1B1 axis plays a crucial role in promoting $\mathrm{CC}$ progression and may be regarded as a novel prognostic marker and possible therapeutic target of CC.

\section{Conclusion}

The importance of piRNA in CC has rarely been tapped. In the present study, piRNA-14633 was found to be significantly upregulated in CCT and CC cells,

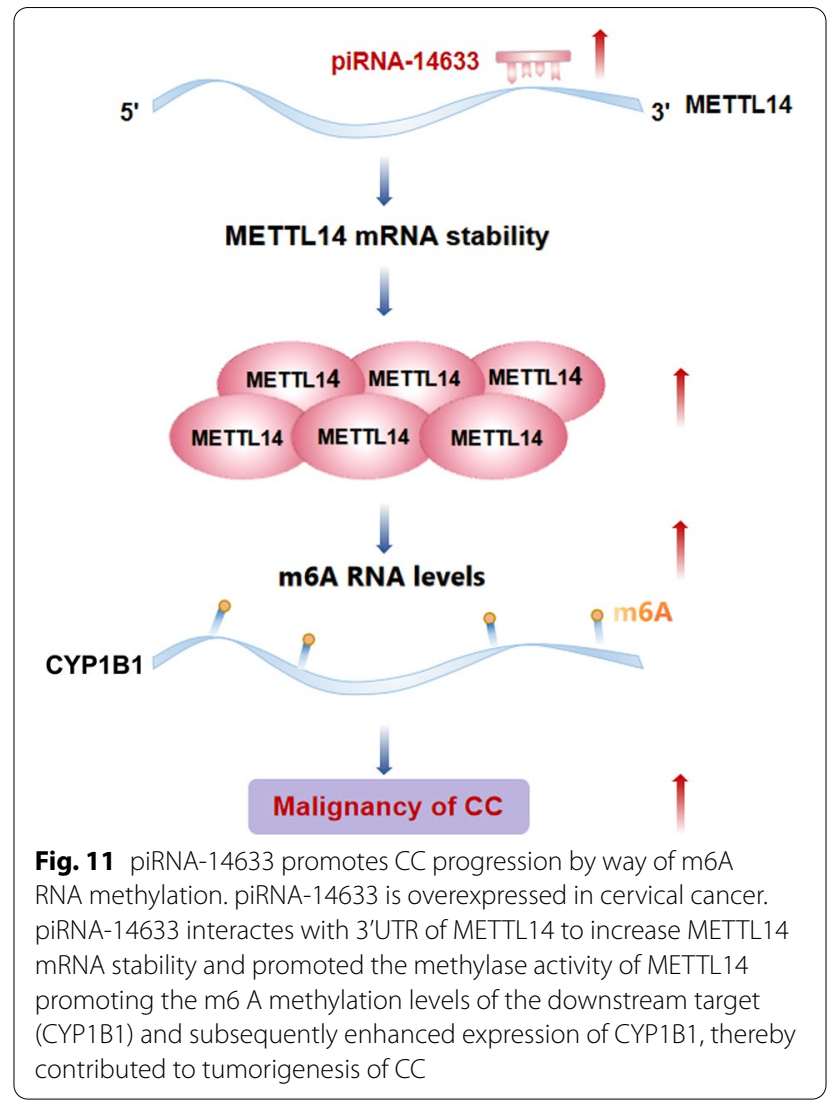

and we also demonstrated that piRNA-14633 contributed to proliferation, migration and invasion by increasing METTL14 expression and m6A RNA methylation. Further studies should follow to understand the deeper mechanism of piRNA-14633 regulating m6A RNA methylation in CC. In short, pi-14633 plays a crucial role in promoting malignancy in $\mathrm{CC}$ via the METTL14/CYP1B1 pathway, suggesting that targeting the piRNA-14633/METTL14/CYP1B1 axis with selective inhibitors could represent a promising therapeutic strategy for treating CC.

\section{Supplementary Information}

The online version contains supplementary material available at https://doi. org/10.1186/s12967-022-03257-2.

Additional file 1: Table S1. Primer sequences for RT-qPCR.

Additional file 2: Fig. S1. Effect of piRNA-14633 mimic/inhibitor on piRNA-14633 expression. a Real-time PCR of piRNA-14633 expression in Caski cells treated with piRNA-14633 mimic or inhibitor. b Real-time PCR of piRNA-14633 expression in Siha cells treated with $50 \mathrm{nM}$ piRNA-14633 mimic or inhibitor. ${ }^{*} P<0.05$ vs. mi-NC group. ${ }^{\sharp} P<0.05$ vs. in-NC group. mi-14633 piRNA-14633 mimic, in-14633 piRNA-14633 inhibitor.

\section{Acknowledgements}

Not applicable. 


\section{Authors' contributions}

TYS and LFK designed the study. ZL and XL collated the data. QX carried out data analyses and produced the initial draft of the manuscript. DW, YZ, and JGZ contributed to drafting the manuscript. SHG, YGY and WYF conducted in vitro experiments. All authors read and approved the final manuscript.

\section{Funding}

The present study was supported by grants from the Medical Science and Technique Foundation of Henan Provincial and ministry co-construction project (grant no. SB201901084), and the Scientific Research Staring Foundation for doctor of Henan Provincial People's Hospital (201701).

\section{Availability of data and materials}

The datasets generated/analyzed during the current study are available.

\section{Declarations}

\section{Ethics approval and consent to participate}

Written informed consent was obtained from all patients prior to the study. Study protocols were approved by Ethics Committee of Henan Provincial People's Hospital and based on the ethical principles for medical research involving human subjects of the Helsinki Declaration. Animal experiments were performed in strict accordance with the recommendations in the Guide for the Care and Use of Laboratory Animals of the National Institutes of Health. The protocol of animal experiments was approved by the Institutional Animal Care and Use Committee of Henan Provincial People's Hospital. Animal experiments were conducted based on minimized animal number and the least pains on experimental animals.

\section{Consent for publication}

All authors consent for publication.

\section{Competing interests}

We declare that we have no conflicts of interest.

\section{Author details}

'Department of Pathology, Henan Provincial People's Hospital, People's Hospital of Zhengzhou University; People's Hospital of Henan University, Zhengzhou, Henan 450003, People's Republic of China. ${ }^{2}$ Henan International Medical Center, Henan Provincial People's Hospital, People's Hospital of Zhengzhou University; People's Hospital of Henan University, Zhengzhou, Henan 450003, People's Republic of China. ${ }^{3}$ Department of Neurologyeurology, Henan Provincial People's Hospital, People's Hospital of Zhengzhou University; People's Hospital of Henan University, Zhengzhou, Henan 450003, People's Republic of China. ${ }^{4}$ Department of Scientific Research and Discipline Construction, Henan Provincial People's Hospital, People's Hospital of Zhengzhou University; People's Hospital of Henan University, Zhengzhou, Henan 450003, People's Republic of China.

Received: 19 July 2021 Accepted: 17 January 2022

Published online: 29 January 2022

\section{References}

1. Sung H, Ferlay J, Siegel RL, Laversanne M, Soerjomataram I, Jemal A, Bray F. Global cancer statistics 2020: GLOBOCAN estimates of incidence and mortality worldwide for 36 cancers in 185 countries. CA Cancer J Clin. 2021;71:209-49.

2. Jiang T, Zhou B, Li YM, Yang QY, Tu KJ, Li LY. ALOX12B promotes carcinogenesis in cervical cancer by regulating the PI3K/ERK1 signaling pathway. Oncol Lett. 2020;20:1360-8.

3. Lyu X, Li G, Qiao Q. Identification of an immune classification for cervical cancer and integrative analysis of multiomics data. J Transl Med. 2021;19:200.

4. Chi RA, van der Watt P, Wei W, Birrer MJ, Leaner VD. Inhibition of Kpnbeta1 mediated nuclear import enhances cisplatin chemosensitivity in cervical cancer. BMC Cancer. 2021:21:106.

5. Zhou J, Zhang Y, Zou X, Kuai L, Wang L, Wang J, Shen F, Hu J, Zhang $X$, Huang $Y$, Chen $Y$. Aberrantly expressed timeless regulates cell proliferation and cisplatin efficacy in cervical cancer. Hum Gene Ther. 2020;31:385-95.

6. Ninova M, Fejes Toth K. New players on the piRNA field. Nat Struct Mol Biol. 2020;27:777-9.

7. Wang X, Lv C, Guo Y, Yuan S. Mitochondria associated germinal structures in spermatogenesis: piRNA pathway regulation and beyond. Cells. 2020;9:1.

8. Rayford KJ, Cooley A, Rumph JT, Arun A, Rachakonda G, Villalta F, Lima MF, Pratap S, Misra S, Nde PN. piRNAs as modulators of disease pathogenesis. Int J Mol Sci. 2021;22:1.

9. Wang $K$, Wang T, Gao XQ, Chen XZ, Wang F, Zhou LY. Emerging functions of piwi-interacting RNAs in diseases. J Cell Mol Med. 2021;25:4893-901.

10. Zeng Q, Wan H, Zhao S, Xu H, Tang T, Oware KA, Qu S. Role of PIWI-interacting RNAs on cell survival: proliferation, apoptosis, and cycle. IUBMB Life. 2020;72:1870-8.

11. Tamtaji OR, Behnam M, Pourattar MA, Hamblin MR, Mahjoubin-Tehran M, Mirzaei $\mathrm{H}$, Asemi Z. PIWI-interacting RNAs and PIWI proteins in glioma: molecular pathogenesis and role as biomarkers. Cell Commun Signal. 2020;18:168.

12. Liu Y, Dou M, Song X, Dong Y, Liu S, Liu H, Tao J, Li W, Yin X, Xu W. The emerging role of the piRNA/piwi complex in cancer. Mol Cancer. 2019:18:123.

13. Guo B, Li D, Du L, ZhuX. piRNAs: biogenesis and their potential roles in cancer. Cancer Metastasis Rev. 2020;39:567-75.

14. Vinasco-Sandoval T, Moreira FC, Pinto P, Santos AM, Cruz RLS, FonsecaCabral G, Anaissi AKM, Lopes KP, Santos A, et al. Global analyses of expressed piwi-interacting RNAs in gastric cancer. Int J Mol Sci. 2020;21:1.

15. Zhong Q, Lu M, Yuan W, Cui Y, Ouyang H, Fan Y, Wang Z, Wu C, Qiao J, Hang J. Eight-IncRNA signature of cervical cancer were identified by integrating DNA methylation, copy number variation and transcriptome data. J Transl Med. 2021;19:58.

16. Lu Y, Chan YT, Tan HY, Li S, Wang N, Feng Y. Epigenetic regulation in human cancer: the potential role of epi-drug in cancer therapy. Mol Cancer. 2020;19:79.

17. Meir Z, Mukamel Z, Chomsky E, Lifshitz A, Tanay A. Single-cell analysis of clonal maintenance of transcriptional and epigenetic states in cancer cells. Nat Genet. 2020;52:709-18.

18. Wang Q, Guo X, Li L, Gao Z, Su X, Ji M, Liu J. N(6)-methyladenosine METTL3 promotes cervical cancer tumorigenesis and Warburg effect through YTHDF1/HK2 modification. Cell Death Dis. 2020;11:911.

19. Li Z, Peng Y, Li J, Chen Z, Chen F, Tu J, Lin S, Wang H. N(6)-methyladenosine regulates glycolysis of cancer cells through PDK4. Nat Commun. 2020;11:2578

20. Wang H, Luo Q, Kang J, Wei Q, Yang Y, Yang D, Liu X, Liu T, Yi P. YTHDF1 aggravates the progression of cervical cancer through m(6)A-mediated up-regulation of RANBP2. Front Oncol. 2021;11:650383.

21. Yi YC, Chen XY, Zhang J, Zhu JS. Novel insights into the interplay between $\mathrm{m}(6) \mathrm{A}$ modification and noncoding RNAs in cancer. Mol Cancer. 2020;19:121.

22. Zhang Y, Geng X, Li Q, Xu J, Tan Y, Xiao M, Song J, Liu F, Fang C, Wang H. m6A modification in RNA: biogenesis, functions and roles in gliomas. J Exp Clin Cancer Res. 2020;39:192.

23. Huang $\mathrm{H}$, Weng $\mathrm{H}$, Chen J. The biogenesis and precise control of RNA m(6)A methylation. Trends Genet. 2020;36:44-52.

24. Gao XQ, Zhang YH, Liu F, Ponnusamy M, Zhao XM, Zhou LY, Zhai M, Liu CY, Li XM, Wang M, et al. The piRNA CHAPIR regulates cardiac hypertrophy by controlling METTL3-dependent N(6)-methyladenosine methylation of Parp10 mRNA. Nat Cell Biol. 2020;22:1319-31.

25. Han H, Fan G, Song S, Jiang YX, Qian CG, Zhang WM, Su Q, Xue XF, Zhuang WZ, Li BZ. piRNA-30473 contributes to tumorigenesis and poor prognosis by regulating $\mathrm{m} 6 \mathrm{~A}$ RNA methylation in DLBCL. Blood. 2021;137:1603-14.

26. Sun T, Wu ZK, Wang XF. LNC942 promoting METTL14-mediated m 6 A methylation in breast cancer cell proliferation and progression. Oncogene. 2020;9:5358-72.

27. Zhu ZM, Huo FC, Pei DS. Function and evolution of RNA N6-methyladenosine modification. Int J Biol Sci. 2020;16:1929-40.

28. He L, Li H, Wu A, Peng Y, Shu G, Yin G. Functions of N6-methyladenosine and its role in cancer. Mol Cancer. 2019;18:176.

29. Shriwas O, Mohapatra P, Mohanty S, Dash R. The impact of m6A RNA modification in therapy resistance of cancer: implication in 
chemotherapy, radiotherapy, and immunotherapy. Front Oncol. 2020;10:612337.

30. Wang X, Lu Z, Gomez A, Hon GC, Yue Y, Han D, Fu Y, Parisien M, Dai Q, Jia $\mathrm{G}$, et al. N6-methyladenosine-dependent regulation of messenger RNA stability. Nature. 2014;505:117-20.

31. Li D, Luo Y, Gao Y, Yang Y, Wang Y, Xu Y, Tan S, Zhang Y, Duan J, Yang Y. piR651 promotes tumor formation in non-small cell lung carcinoma through the upregulation of cyclin D1 and CDK4. Int J Mol Med. 2016;38:927-36.

32. Ding $X$, Li Y, Lu J, Zhao Q, Guo Y, Lu Z, Ma W, Liu P, Pestell RG, Liang C, Yu Z. piRNA-823 is involved in cancer stem cell regulation through altering DNA methylation in association with luminal breast cancer. Front Cell Dev Biol. 2021;9:641052.

33. Fu A, Jacobs $D I$, Zhu Y. Epigenome-wide analysis of piRNAs in genespecific DNA methylation. RNA Biol. 2014;11:1301-12.

34. Zoch A, Auchynnikava T, Berrens RV, Kabayama Y, Schopp T, Heep M, Vasiliauskaite L, Perez-Rico YA, Cook AG, Shkumatava A, et al. SPOCD1 is an essential executor of piRNA-directed de novo DNA methylation. Nature. 2020;584:635-9.

35. Nagamori I, Kobayashi H, Nishimura T, Yamagishi R, Katahira J, KuramochiMiyagawa S, Kono T, Nakano T. Relationship between PIWIL4-mediated H3K4me2 demethylation and piRNA-dependent DNA methylation. Cell Rep. 2018;25:350-6.

36. Weng W, Liu N, Toiyama Y, Kusunoki M, Nagasaka T, Fujiwara T, Wei Q, Qin $\mathrm{H}$, Lin $\mathrm{H}, \mathrm{Ma}$ Y, Goel A. Novel evidence for a PIWI-interacting RNA (piRNA) as an oncogenic mediator of disease progression, and a potential prognostic biomarker in colorectal cancer. Mol Cancer. 2018;17:16.

37. Huang $H$, Weng $H$, Sun W, Qin X, Shi H, Wu H, Zhao BS, Mesquita A, Liu C, Yuan CL, et al. Recognition of RNA N(6)-methyladenosine by IGF2BP proteins enhances mRNA stability and translation. Nat Cell Biol. 2018;20:285-95.

38. Pan J, Xu L, Pan H. Development and validation of an m6A RNA methylation regulator-based signature for prognostic prediction in cervical squamous cell carcinoma. Front Oncol. 2020;10:1444.

39. Ma X, Li Y, Wen J, Zhao Y. m6A RNA methylation regulators contribute to malignant development and have a clinical prognostic effect on cervical cancer. Am J Transl Res. 2020;12:8137-46.

40. Zou D, Dong L, Li C, Yin Z, Rao S, Zhou Q. The m(6)A eraser FTO facilitates proliferation and migration of human cervical cancer cells. Cancer Cell Int. 2019;19:321.

41. Alshammari FOFO, Al-Saraire YM, Youssef AMM, Alrawashdeh HM Cytochrome P450 1B1 overexpression in cervical cancers: cross-sectional study. Interact J Med Res. 2021;10:e31150.

\section{Publisher's Note}

Springer Nature remains neutral with regard to jurisdictional claims in published maps and institutional affiliations.

Ready to submit your research? Choose BMC and benefit from:

- fast, convenient online submission

- thorough peer review by experienced researchers in your field

- rapid publication on acceptance

- support for research data, including large and complex data types

- gold Open Access which fosters wider collaboration and increased citations

- maximum visibility for your research: over 100M website views per year

At BMC, research is always in progress.

Learn more biomedcentral.com/submissions 